\title{
First Report of ethnobotanical studies of tehsil Noorpur Thal, District Khushab, Punjab, Pakistan
}

\section{Zaheer Yousaf}

\author{
zaheeryousaf56@yahoo.com
}

\begin{abstract}
The study is based on gathering of information by interviewing villagers, herbalists, hakims and farmers, following a preset list of questions followed by analysis of the data collected. Plant samples were gathered and their morphological characteristics described. Their various uses including medicinal uses, where reported were gathered. The present work is a significant contribution to the existing knowledge because ethno botany as a interdisciplinary science understanding oflocal social dynamics, institutions and different values attributed to resources. These values may be symbolic, religious or political for a given society, while same plant resources may represent only an economic value for our social group. Fuel woodneeds are also met by collecting dried fodder branches, by products of crops and dried animal dung. It is recommended that botanical ganders of medicinal plants should be established. Data was analyzed at $P(0.05-5 \%)$.

Keywords- Interviewing Villagers, Medicinal Uses, Ethno botany, Plant Samples, Conservational Reserves, Botanical Garden, Products of Crops and Animal Dung.
\end{abstract}

\section{INTRODUCTION}

From ancient Time, plants are being used in various diseases. Many of today's drugs have been derived from plant sources. Pharmacognosy is the study of medicinal and toxic products from natural plant sources. More than six thousand species are used as medicines drives from medicinal plant are $\$ 43$ billion. More than $75 \%$ of Pakistani population depended on tradition medicines for all are most of its medicinal needs. Ethnoparmacological study not only envisage the possibility of identifying new herbal drug, but also bring on record the hidden knowledge confied to traditional society all over the world (Leporatti 1990) bhattari, 1992 padhye at al 1992 , yang 1992, omino et al, 1993, Gils et al, 1994, bhanday et al, 1997, Verma et al, 1998).

\section{Study Area}

The very word Khushab, derived from two Persian words
Khush" and "Aab" meaning good or peasant potable water. District Khushab is one of the four districts of Sargodha Division. The district lies between north latitude 31-33 to 32-43 degree and east longitude 71-35 to 72-37 degrees. The average length of the District from north to south is about 116 Kilometers: while its breadth from east to west is about 56 kilometer. The District comprises an area of $1,627,688$ acres or 6,511 sq. Kilometer.

The few trees to be found in the dry and sandy that are chiefly Jund, (Prosopis cineraria (L) Druce), which is found in graves protected by the reputation of some departed scunt: stunted kikar, rarely found the round ponds and a grave of beri (ZizyphusnummulariaBurm.f.) trees found the town ofNurpur, which are specially protected by a clause in the village administration paper. The characteristic bushes of the region are the lana (salaola), akk (Calotropis pro cera R. Br.) and harmal (paganumharmala 1.) which have alteady been described and the phoy (Calligounmpolygonoidea Linn.) a good fodder plant, little found except in RakhNurpur, but (Pauderia pilosa) a low whitish plant with flower heads like catkins, khipp, (Crotalaria burhia) some time used for making ropes for temporary use and summa and karturnma (Citrulus colosynthis (L.) Shrad.) with its trailing stems and beautiful green and yellow orange likes fruit scattered in the profusion over the sandy hills. Their taste is very bitter, but goats eat them and medicine for horses is prepared from them to prevent indigestion.

In the past only cultivation consisted of small patches of cheap millets and pulses, or very inferior watermelons. But it has since been discovered that excellent grass of crops can be raised in an ordinary winter and year by year larger areas are devoted to raising them, the change from pasture to agriculture as the principal means of livelihood is going on apace. The resulting development of land is, of course, over-shadowed by the brilliant success of the lower Jhelum Canal, but is nonetheless remarkable. 
In the flooded lands along the rivers lei or pilchi

(Tamarix dioica Roxb. Ex Royh) springs up considerable thickets and is used for wattling, baskets and roofs. The akk (Calotropis procera $\mathrm{R}$. Br.) is very common in sandy soil. It is also useful for snakebite (Ajibade et al., 2005).

\section{METHODOLOGY}

The survey was conducted from March, 2003 to February 2006. The methodology was based on interviews using checklist and questionnaire of information (Martin, 1995). The interviewees in the villages were chosen at random. Total No of interviewees conducted are 750 consists of 400 males and 350 females. The interviewees were landholders (zamindars), Agriculturists, pansars, Hakims and Farmers, and most of them were mainly graduates and Government employee. In the first step, detailed knowledge about the local and indigenous people was collected. A regional study on the epidemiology tradition medicines and ecology of the people and their environment was prepared. In order to prioritize plant collectors, a number of international data basis were searched to obtain all the relevant ethno-medical, biological and chemical information on the plant known to be used in that region.

Following parameters were adopted for the study:

\section{A. ETHNOMEDICINAL USES}

". Uses of herbal medicinal

". Parts of the plants used

'- Ailments treated

$\therefore$ Success of use

Source of supply

Average annual stock (quantity)

- Average annualsale (quantity)

. Types of people treated

No. of people treated per day

Trend in use of medicinal plants

\section{B. FODDER USES}

$i$, Fodder priority

,. Fodder effects

Animal types

. Preferred pats

\section{ETHNOBOTANICAL USES}

- Vegetables and pot herbs

. Fruit yielding

- Poisonous plants

- Method of use

- Prices per KG.

Plant grown/cultivated

- Plant material stored

- Quantity sold per year

.' Sold in the form (dry/fresh)

- Used in the form (dry/fresh)

!. Total number of species traded

- Harvesting season

- Method of preparation (infused/boiled/distilled/fresh juice)

- Details of preparation Method of internal application (infusion/decoction/syrup chewed) Method of external application (poultice, fixed oil, lotion cream)

Age groups of people using the species. Health maintenance

Types of livestock treated Livestock ailment treated Use of herbs in combination with other herbs

Period of storage of plants/herbs 
Processing Additive used

Domestic, community-wise and market value Species preferred for sale

Average price per unit

Source of fuel for domestic purpose_

Average consumption of fuel per day for each household

D. FUEL SOURCES AND ITS CONSUMPTION

- Source of fuel for domestic purpose

- Average consumption of fuel day for each household

- Fuel types (i. e. fuel-wood, kerosene oil, LPG, crop residues, cow-dung, wood-waste, charcoal)

- Average monthly fuel requirement in summer and winter seasons
- $\quad$ Variation of fuel requirement in summer and winter seasons

- Dependency of people on wood fuel

- Prices of different types of fuels

- Availability of wood fuel

- $\quad$ Source of wood fuel

- Main cases of non-availability of fuel wood fuel

- $\quad$ Species used for wood fuel

- $\quad$ Preferred species of wood fuel

- $\quad$ Estimated percentage increase of trees III the surrounding

- $\quad$ Source of information about trees

- $\quad$ Suggestion to increase tree cover in the area

Table.1: Species used for different ailments

\begin{tabular}{|c|c|c|c|c|c|}
\hline S.No & $\begin{array}{l}\text { Name of } \\
\text { species }\end{array}$ & Scientific Name & Part used & Illness & Success \\
\hline 1. & AK & $\begin{array}{l}\text { Calotropis } \\
\text { procera } \mathrm{R} . \mathrm{Br} .\end{array}$ & Stem & Joint Pain & Comforts \\
\hline 2. & Harmal & $\begin{array}{l}\text { Peganum } \\
\text { harmala } \mathrm{L} .\end{array}$ & Seed & Abdominal Pain & Comforts \\
\hline 3. & Akashbel & $\begin{array}{l}\text { Cuscuta reflexa } \\
\text { Roxb. }\end{array}$ & Stem & Phorey & Comforts \\
\hline 4. & Tumma & $\begin{array}{l}\text { Citrullus } \\
\text { colocynthus (L.) } \\
\text { Schard }\end{array}$ & $\begin{array}{l}\text { Seed Fruit } \\
\text { Root } \\
\text { Oil of seed } \\
\text { Root }\end{array}$ & $\begin{array}{l}\text { 1. } \text { Constipation } \\
\text { 2. Stomach ailment } \\
\text { 3. Immunity for Rani Khet } \\
\text { 4. Diseases } \\
\text { 5. Abn stroke/Heat } \\
\text { 6. Ammnorrhea } \\
\text { 7. Ascites } \\
\text { 8. Asthma } \\
\text { 9. Billousnes } \\
\text { 10. Cerebral congestion } \\
\text { 11. Elephantiasis } \\
\text { 12. Epilepsy } \\
\text { 13. Facial paralysis } \\
\text { 14. Fever } \\
\text { 15. Gout } \\
\text { 16. Hepaticcongestion } \\
\text { 17. Jaundice } \\
\text { 18. Leprosy } \\
\text { 19. Liver dibility } \\
\text { 20. Neuralgic complication } \\
\text { 21. Paralysis }\end{array}$ & $\begin{array}{ll}\text { 1. } & \text { Comforts } \\
\text { 2. } & \text { Very Effective } \\
\text { 3. } & \text { Very Effective } \\
\text { 4. } & \text { Cold effect }\end{array}$ \\
\hline
\end{tabular}




\begin{tabular}{|c|c|c|c|c|c|}
\hline & & & $\begin{array}{l}\text { Root } \\
\text { Poultice of } \\
\text { root Juice } \\
\text { Oil of Seed }\end{array}$ & $\begin{array}{l}\text { 22. Rheumatism } \\
\text { 23. Sciatica } \\
\text { 24. Visceral congestion } \\
\text { 25. Inflamation of breast } \\
\text { 26. Remedy of dropsy Snake } \\
\text { bites scorpion stings and } \\
\text { bowl complatints } \\
\text { (dysentery, diarrhea) } \\
\text { Epilepsy and for growth } \\
\text { and blackening of hairl }\end{array}$ & \\
\hline 5. & $\begin{array}{l}\text { Khoob Klan } \\
\text { (Chuniakha) }\end{array}$ & $\begin{array}{l}\text { Sisymbrium irio } \\
\text { Crantz ex Steud }\end{array}$ & Seed & $\begin{array}{ll}\text { 1. } & \text { Typhoid } \\
\text { 2. } & \text { Small Pox (Chechak) } \\
\text { 3. } & \text { Chest debility, } \\
& \text { cholera,cough, fever, } \\
& \text { harassness vocalorgan } \\
& \text { debility, vomiting }\end{array}$ & $\begin{array}{l}\text { Removes Small Pox } \\
\text { (chechak grains) }\end{array}$ \\
\hline 6. & Saunf & $\begin{array}{l}\text { Foeniculum } \\
\text { vulgare Miller }\end{array}$ & Whole Plant & $\begin{array}{ll}\text { 1. } & \text { Digestion problem } \\
\text { 2. } & \text { Gas Trouble } \\
\text { 3. } & \text { Female illness } \\
\text { 4. } & \text { Nervous disease }\end{array}$ & $\begin{array}{ll}\text { 1. } & \text { Increases } \\
& \text { Digestion } \\
\text { 2. } & \text { Gas trouble } \\
& \text { recovers. } \\
\text { 3. } & \text { Treatment. } \\
\text { 4. } & \text { Comforts. }\end{array}$ \\
\hline 7. & HarniKaKhaj & $\begin{array}{l}\text { Cistanchetabulos } \\
a \text { Wight }\end{array}$ & Whole Plant & Blood Purifier & Comforts \\
\hline 8. & Jawah & $\begin{array}{l}\text { Carum copticum } \\
\text { Benth }\end{array}$ & Whole Plant & Blood Purifier & Comforts \\
\hline 9. & Boophali & $\begin{array}{l}\text { Corchorusaes } \\
\text { tuans Linn. }\end{array}$ & Whole Plant & Stomach and liver heat & $\begin{array}{l}\text { Patient becomes } \\
\text { healthy }\end{array}$ \\
\hline 10. & Lauhurian & $\begin{array}{l}\text { Tecomella } \\
\text { undulate }\end{array}$ & Whole Plant & Defect in Uterus & $\begin{array}{l}\text { Patient becomes } \\
\text { healthy }\end{array}$ \\
\hline 11. & Kahnu & & Whole Plant & Defect in Uterus & $\begin{array}{l}\text { Patient becomes } \\
\text { healthy }\end{array}$ \\
\hline 12. & Bhakra & $\begin{array}{l}\text { Tribulus } \\
\text { camaldulensis. L }\end{array}$ & Seed & $\begin{array}{l}\text { Gall Bladder illness, Kidney } \\
\text { Allergy }\end{array}$ & Most successful \\
\hline 13. & Boophali & $\begin{array}{l}\text { Corchorus } \\
\text { aestuans Linn. }\end{array}$ & Whole Plant & $\begin{array}{l}\text { 1. Maleness in Man } \\
\text { 2. Liqueria }\end{array}$ & Successful \\
\hline 14. & Hareer/Arhar & Cajanuscajan L. & Root & Spermatorrhoea & Successful \\
\hline 15. & $\begin{array}{l}\text { Asgandh/IksinN } \\
\text { eelwat }\end{array}$ & $\begin{array}{l}\text { Withania } \\
\text { somnifera L. }\end{array}$ & $\begin{array}{l}\text { 1. Root } \\
\text { Decoction }\end{array}$ & $\begin{array}{l}\text { 1. Weakness of sexual } \\
\text { organ. } \\
\text { 2. Premature ejaculation } \\
\text { 3. Leucorrhoea and frequent } \\
\text { miscarriage (ladies) } \\
\text { 4. Emaciation (women and } \\
\text { children) } \\
\text { 5. General debility } \\
\text { 6. Glandular swelling } \\
\text { 7. Leucoderrma }\end{array}$ & Successful \\
\hline
\end{tabular}




\begin{tabular}{|c|c|c|c|c|c|}
\hline & & & $\begin{array}{l}\text { 2. Root } \\
\text { Paste and } \\
\text { Cow Urine }\end{array}$ & $\begin{array}{l}\text { 8. Loss of memory } \\
\text { 9. Nervus exhaustion } \\
\text { 10. Rhumatica affection. } \\
\text { 11. Snile debility } \\
\text { 12. Syphilis } \\
\text { 13. Skin diseases }\end{array}$ & \\
\hline 16. & Puthkanda & $\begin{array}{l}\text { Achyrandthes } \\
\text { aspera } \mathrm{L} \text {. }\end{array}$ & Root & 14. Impotency & Successful \\
\hline 17. & Bathu & $\begin{array}{l}\text { Chenopodium } \\
\text { album } \mathrm{L} \text {. }\end{array}$ & $\begin{array}{l}\text { 1. Cooked } \\
\text { leaves . } \\
\text { 2. Leaf } \\
\text { extract } \\
\text { 3. Root } \\
\text { powder }\end{array}$ & $\begin{array}{l}\text { 1. Urinary troubles and colic } \\
\text { 1. Piles } \\
\text { 2. Cought } \\
\text { 3. Worms } \\
\text { 1. Spermatorrhoea }\end{array}$ & Successful \\
\hline 18. & Drunk & $\begin{array}{l}\text { Polygonum } \\
\text { plebijum } \mathrm{R} . \mathrm{Br} .\end{array}$ & $\begin{array}{l}\text { 1. Plant } \\
\text { Decoction } \\
\text { 1. Plant ash } \\
+ \text { Oil }\end{array}$ & $\begin{array}{l}\text { 1. Colic complaints } \\
\text { 1. Eczema }\end{array}$ & Successful \\
\hline 19. & Jau & $\begin{array}{l}\text { Hordeum } \\
\text { vulgare Linn. }\end{array}$ & Leaf Juice & Cataract & Successful \\
\hline 20. & Jund & $\begin{array}{l}\text { Prosopis } \\
\text { cineraria (Linn) } \\
\text { Druce }\end{array}$ & Leaves & Leucorrhoea & Successful \\
\hline 21. & $\begin{array}{l}\text { Mako/MirchBo } \\
\text { oti }\end{array}$ & $\begin{array}{l}\text { Solanumnigrum } \\
\text { L. }\end{array}$ & $\begin{array}{l}\text { 1. Leaf paste } \\
\text { and } \\
\text { branches } \\
\text { 2. Whole } \\
\text { plant } \\
\text { Decoction }\end{array}$ & $\begin{array}{l}\text { 1. Jaundice } \\
\text { 2. High fever } \\
\text { 1. Spermatorrhoea }\end{array}$ & Successful \\
\hline 22. & Kashmiri Kiker & $\begin{array}{l}\text { Prosopis julflora } \\
\text { Swartz }\end{array}$ & & & \\
\hline 23. & Pilchi/Lei/Frash & $\begin{array}{l}\text { TamarixdlocaRo } \\
\text { xb. ex Roth }\end{array}$ & $\begin{array}{l}\text { 1. Bark } \\
\text { (Bitter and } \\
\text { Tonic) }\end{array}$ & $\begin{array}{l}\text { 1. Annal Fisher } \\
\text { 2. Cough } \\
\text { 3. Diarrhoea } \\
\text { 4. Dysentry } \\
\text { 5. Pectrol Affection } \\
\text { 6. Piles } \\
\text { 7. Uleers } \\
\text { 8. Leucorrhoea } \\
\text { 9. Spoleen Trouble } \\
\text { 10. Leucoderrma }\end{array}$ & \\
\hline 24. & Chiraita & Swertia chiraita & $\begin{array}{l}\text { Whole plant } \\
\text { and } \\
\text { Decoction }\end{array}$ & $\begin{array}{l}\text { All kinds of fever particularly } \\
\text { (i) Pneumonia } \\
\text { (ii) Malaria } \\
\text { (iii) Typhoid }\end{array}$ & \\
\hline
\end{tabular}

Table.2: Ethno-botanical uses of different plant species 


\begin{tabular}{|c|c|c|c|c|c|c|}
\hline $\begin{array}{l}\text { S. } \\
\text { No }\end{array}$ & Local Name & Scientific Name & Part Used & Fuel Wood & Timber & Fodder \\
\hline 1. & Shrin & Albizia lebbek (L.) Willd & & - & & Fodder \\
\hline 2. & Kiker & Acacia nilotica $\mathrm{L}$. & $\begin{array}{l}\text { Leaves \& } \\
\text { Wood }\end{array}$ & $\begin{array}{l}\text { Excellent } \\
\text { Fuel }\end{array}$ & $\begin{array}{l}\text { Agricultural } \\
\text { implements }\end{array}$ & - do- \\
\hline 3. & Beri & Zizyphus numularia (Burm.f.) & Leaves & - & - & -do- \\
\hline 4. & Shisham & Dalbergia sisoo Roxb. Ex DC. & Leaves & - & - & -do- \\
\hline 5. & Khagal & Tamarix dioica Roxb. ex Roth & Stem & Fuel & Building & - \\
\hline 6. & Sufeda & Eucalyptus blobulus & - & - & Building & - \\
\hline 7. & Channa & Cicer arietinum $\mathrm{L}$. & Seed \& Stem & Fuel & - & Fodder \\
\hline 8. & KhoobKalan & $\begin{array}{l}\text { Sisymbrium irio Cranz ex } \\
\text { Stued }\end{array}$ & - & - & - & -do- \\
\hline 9. & Jund & Prosopis cineraria (L.) Druce) & Stem & Fuel & & -do- \\
\hline 10. & Gowara & Cyamopsis tetragonoloba $\mathrm{L}$. & Stem & Fuel & - & -do- \\
\hline 11. & Burs in & Trifolium repens $\mathrm{L}$. & Except roots & - & - & -do- \\
\hline 12. & Jowar & $\begin{array}{l}\text { Sorghum bicolor (Linn.) } \\
\text { Moench }\end{array}$ & Except roots & - & - & -do- \\
\hline 13. & Bajra & $\begin{array}{l}\text { Pennisetum typhoideum } \\
\text { (Burm. F.) Staff \& Hubbard }\end{array}$ & Except roots & - & - & -do- \\
\hline 14. & Loosen & Trifolium alexandrianum $\mathrm{L}$. & Except roots & - & - & -do- \\
\hline 15 & Jowadar & Avena sativa Linn. & Except roots & - & - & -do- \\
\hline 16 & $\begin{array}{l}\text { Kashmiri } \\
\text { Kiker }\end{array}$ & Prosopis julifora (Sw.) DC. & Except roots & Fuel & Construction & $\begin{array}{l}\text { Fodder paper and } \\
\text { consmeticindustry } \\
\text { industry }\end{array}$ \\
\hline 17 & $\begin{array}{l}\text { Wheat } \\
\text { (Kanak) }\end{array}$ & Triticum aestivum Linn. & Hay/Stem & - & - & \\
\hline 18 & Sarsoon & Brassica comperis $\mathrm{L}$. & Stem/Leaves & - & - & -do- \\
\hline 19 & Kallar grass & Leptochola fusca & Stem/Leaves & - & - & -do- \\
\hline 20 & Juo & Hordeum vulgare Linn. & Upper part & - & - & -do- \\
\hline 21 & Gana & Saccharum spontaneum Linn. & $\begin{array}{l}\text { 1. Stem } \\
\text { 2. Thin end of } \\
\text { the stock (Tili) } \\
\text { 3. Shea thing } \\
\text { petiole after } \\
\text { being burnt at } \\
\text { the lower end } \\
\text { beaten with a } \\
\text { mallet yield a } \\
\text { fiber (Munj) }\end{array}$ & & $\begin{array}{l}\text { 1. Thatching } \\
\text { and making } \\
\text { chairs } \\
\text { 2. Making } \\
\text { baskets and } \\
\text { screens (sirki) } \\
\text { 3. Munj is } \\
\text { twisted into } \\
\text { ropes }\end{array}$ & \\
\hline 22 & Kah & Saccharum spontaneum Linn & & & & $\begin{array}{l}\text { 1. Grazing for } \\
\text { Buflalos } \\
\text { 2. Making brushes } \\
\text { 3. Use to strew on the } \\
\text { floors of mosques }\end{array}$ \\
\hline 23 & Dhub & $\begin{array}{l}\text { Desmostachya bipinnata (L.) } \\
\text { stapf }\end{array}$ & & & & Making ropes \\
\hline
\end{tabular}




\begin{tabular}{|c|c|c|c|c|}
\hline S.No & Name of Species & Local Name & Qty (Kg) & Price (Rs.) \\
\hline 1. & Peganum harmalaL. & Harmel & 1 & 50 \\
\hline 2. & Cusuta reflexa Roxb. & Ahashbel & 1 & 500 \\
\hline 3. & Citrulluscolocynthus (L.) Schrad & Tumma & 1 & 50 \\
\hline 4. & Tribulus camaldulensis $\mathrm{L}$. & Bhakra & 1 & 100 \\
\hline 5. & Calotropis procera $\mathrm{R} . \mathrm{Br}$. & $\mathrm{Ak}$ & 1 & 1000 \\
\hline 6. & Eucayptus globules & Sufeda & 40 & $110 /(2.7 / \mathrm{K} . \mathrm{g})$ \\
\hline 7. & Tamarix dioica Roxb. ex Roth & Khagal & 40 & $100(2.5 / \mathrm{kg})$ \\
\hline 8. & Dalbergia sissoo Roxb. ex DC. & Shishum & 40 & $200(5 / \mathrm{kg})$ \\
\hline 9. & Citrulus colocynthus (L.) Schrad. & Tumma & 1 & 15 \\
\hline 10 & Trianthma portulacastrum $\mathrm{L}$. & Biskhapra & 1 & 30 \\
\hline 11. & Acacia nilotica $\mathrm{L}$ & GondKiker & 1 & 40 \\
\hline 12. & Sisymbriumirio Crantz.ex Steud & $\begin{array}{l}\text { KhoobKalan } \\
\text { (Chaniakha) }\end{array}$ & 1 & 50 \\
\hline 13. & Cicer arietinum $\mathrm{L}$. & Channa (Black) & 1 & 20 \\
\hline 14. & Cicer arietinum $\mathrm{L}$. & Channa (White) & 1 & 40 \\
\hline 15. & Foeniculum vulgare Miller & Sounf & 1 & 20 \\
\hline 16. & Zizyphus nummularia (Burm.f.) & Beri & 1 & 3 \\
\hline 17. & - & Lahurian & 1 & $1500-28000$ \\
\hline 18. & Corchorus astuans Linn. & Boophali & 1 & 65 \\
\hline 19. & Peganum harmala $\mathrm{L}$. & Harmel & 1 & 40 \\
\hline 20. & Tribulus calendulensis $\mathrm{L}$. & Bhakra & 1 & 40 \\
\hline 21. & Plantago psilliumForssk. & Isbaghol & 1 & 80 \\
\hline 22. & Tribulus cameldulensis $\mathrm{L}$. & Bhakra & 1 & 20 \\
\hline 23. & Corchorus astuans Linn. & Boophali & 1 & 150 \\
\hline
\end{tabular}

\section{$\underline{\text { Statistical Analysis }}$}

Table.1: Variation in the price offuel yielding species by using ANOVA Statistical Analysis of the price offuel yielding species of Thal

\begin{tabular}{|c|c|c|c|c|}
\hline \multirow{3}{*}{} & \multicolumn{2}{|c|}{ Prices } & \multicolumn{2}{c|}{ Total } \\
\cline { 2 - 5 } & 50 & 40 & 20 & 110 \\
\cline { 2 - 5 } & 50 & 50 & 40 & 75 \\
\cline { 2 - 5 } & 15 & 20 & 40 & 110 \\
\cline { 2 - 5 } & 30 & 40 & $\mathbf{1 6 5}$ & $\mathbf{4 6 0}$ \\
\hline TOTAL: & $\mathbf{1 4 5}$ & $\mathbf{1 5 0}$ & & \\
\hline
\end{tabular}

\begin{tabular}{|c|c|c|c|c|}
\hline Source of variation & Sum of Squares & Degree of Freedom & Mean Squares & Computed Frequency \\
\hline Row means & 1383 & 3 & 461 & F1=2.82 \\
\hline Column means & 54 & 2 & 27 & F2=.165 \\
\hline Errors & 980 & 6 & 163 & \\
\hline Total & 2417 & 11 & & \\
\hline
\end{tabular}

Non-significant

Table.2: Variation of average monthly wood-waste fuel in summer and winter

\begin{tabular}{|c|c|c|c|}
\hline \multicolumn{5}{|c|}{ WINTER } \\
\hline $\mathrm{X} 1$ & $\mathrm{X}^{2} 1$ & $\mathrm{X} 2$ & $\mathrm{X}^{2} 2$ \\
\hline 40 & 1600 & 80 & \multicolumn{2}{c|}{6400} \\
\hline
\end{tabular}




\begin{tabular}{|c|c|c|c|}
\hline 40 & 1600 & 80 & 6400 \\
\hline 40 & 1600 & 80 & 6400 \\
\hline 80 & 6400 & 1600 & 25600 \\
\hline 40 & 1600 & 80 & 6400 \\
\hline 80 & 6400 & 1600 & 25600 \\
\hline 80 & 6400 & 1600 & 25600 \\
\hline 40 & 1600 & 80 & 6400 \\
\hline
\end{tabular}

At $5 \%$ Significant

Table.3: Variation of Fuel Wood Consumption in summer and Winter Seasons

\begin{tabular}{|c|c|c|c|}
\hline \multicolumn{2}{|c|}{ WINTER } & \multicolumn{2}{c|}{ SUMMER } \\
\hline $\mathrm{X} 1$ & $\mathrm{X}^{2} 1$ & 60 & 6400 \\
\hline 120 & 14400 & 600 & 360000 \\
\hline 800 & 640000 & 200 & 40000 \\
\hline 240 & 576000 & 400 & 160000 \\
\hline 600 & 360000 & 40 & 1600 \\
\hline 80 & 6400 & 200 & 40000 \\
\hline 240 & 56700 & 40 & 1600 \\
\hline 400 & 160000 & 80 & 6400 \\
\hline 120 & 14400 & 80 & 6400 \\
\hline 94 & 8836 & 120 & 14400 \\
\hline 174 & 30276 & 94 & 8836 \\
\hline 147 & 21609 & 80 & 6400 \\
\hline 120 & 14400 & 80 & 6400 \\
\hline 120 & 14400 & 100 & 10000 \\
\hline 100 & 10000 & 80 & 6400 \\
\hline 120 & 144000 & S & \\
\hline
\end{tabular}

At $5 \%$ Significant

Table.4: Variations in the kerosene Oil consumption in the summer and Winter

\begin{tabular}{|c|c|c|c|}
\hline \multicolumn{2}{|c|}{ SUMMER } & \multicolumn{2}{c|}{ WINTER } \\
\hline X1 & $X^{2}$ & 25 & 625 \\
\hline 20 & 400 & 25 & 625 \\
\hline 20 & 400 & 15 & 225 \\
\hline 10 & 100 & 15 & 225 \\
\hline 10 & 100 & 4 & 16 \\
\hline 2 & 4 & 2 & 4 \\
\hline 1 & 1 & 2 & 4 \\
\hline 1 & 1 & 4 & 16 \\
\hline 2 & 4 & 15 & 225 \\
\hline 10 & 100 & 15 & 225 \\
\hline 10 & 100 & 15 & 225 \\
\hline
\end{tabular}




\begin{tabular}{|c|c|c|c|}
\hline 60 & 3600 & 15 & 225 \\
\hline 60 & 3600 & 15 & 225 \\
\hline
\end{tabular}

At $5 \%$ Significant

Table.5: Variations in the Wood waste consumption in the summer and Winter

\begin{tabular}{|c|c|c|c|}
\hline \multicolumn{2}{|c|}{ SUMMER } & \multicolumn{2}{|c|}{ WINTER } \\
\hline $\mathrm{X} 1$ & $\mathrm{X}^{2} 1$ & $\mathrm{X} 2$ & $\mathrm{X}^{2} 2$ \\
\hline 40 & 1600 & 80 & 6400 \\
\hline 40 & 1600 & 80 & 6400 \\
\hline 40 & 1600 & 80 & 6400 \\
\hline 80 & 6400 & 160 & 25600 \\
\hline 80 & 6400 & 40 & 1600 \\
\hline 40 & 1600 & 80 & 6400 \\
\hline $\mathbf{8 0}$ & 6400 & 40 & 1600 \\
\hline 40 & 1600 & 80 & 6400 \\
\hline 94 & 8836 & 80 & 6400 \\
\hline 174 & 30276 & 120 & 14400 \\
\hline 147 & 21609 & 94 & 8836 \\
\hline 120 & 14400 & 80 & 6400 \\
\hline 120 & 14400 & 80 & 6400 \\
\hline 100 & 10000 & 100 & 10000 \\
\hline 120 & 14400 & 80 & 6400 \\
\hline
\end{tabular}

At $5 \%$ Significant

Table.6: Variations in LPG consumption in the summer and Winter

\begin{tabular}{|c|c|c|c|}
\hline \multicolumn{2}{|c|}{ SUMMER } & \multicolumn{2}{c|}{ X 2 X } \\
\hline X1 & $X^{2} 1$ & $\mathbf{8 0 0} 2$ \\
\hline 20 & 400 & $\mathbf{8 0 0}$ & $\mathbf{6 4 0 0}$ \\
\hline 20 & 400 & 2 & 4 \\
\hline 10 & 100 & 2 & 4 \\
\hline 10 & 100 & 2 & 4 \\
\hline 2 & 4 & 4 & 16 \\
\hline 1 & 1 & 15 & 225 \\
\hline 2 & 4 & 15 & 225 \\
\hline 10 & 100 & 15 & 225 \\
\hline 10 & 100 & 15 & 225 \\
\hline 60 & 100 & 15 & 225 \\
\hline 60 & 3600 & 15 & 225 \\
\hline
\end{tabular}

At 5\% Significant 
Table.7: Variations in the Crop-residues consumption in the summer and Winter

\begin{tabular}{|c|c|c|c|}
\hline \multicolumn{2}{|c|}{ SUMMER } & \multicolumn{2}{c|}{ WINTER } \\
\hline$X 1$ & $X^{2} 1$ & $X 2$ & $X^{2} 2$ \\
\hline 120 & $\mathbf{1 4 4 0 0}$ & $\mathbf{1 6 0}$ & $\mathbf{2 5 6 0 0}$ \\
\hline 120 & $\mathbf{1 4 4 0 0}$ & $\mathbf{1 6 0}$ & $\mathbf{2 5 6 0 0}$ \\
\hline 40 & $\mathbf{1 6 0 0}$ & $\mathbf{8 0}$ & $\mathbf{6 4 0 0}$ \\
\hline 400 & $\mathbf{1 6 0 0 0 0}$ & $\mathbf{6 0 0}$ & $\mathbf{3 6 0 0 0 0}$ \\
\hline 80 & $\mathbf{6 4 0 0}$ & $\mathbf{1 2 0}$ & $\mathbf{4 4 0 0}$ \\
\hline 20 & $\mathbf{4 0 0}$ & $\mathbf{2 0}$ & $\mathbf{2 5 6 0 0}$ \\
\hline $\mathbf{8 0}$ & $\mathbf{6 4 0 0}$ & $\mathbf{2 0}$ & $\mathbf{4 0 0}$ \\
\hline 10 & $\mathbf{1 0 0}$ & & \\
\hline
\end{tabular}

At 5\% Significant

Table.8: Variations in the Cow-dung consumption in the summer and Winter

\begin{tabular}{|c|c|c|c|}
\hline \multicolumn{2}{|c|}{ SUMMER } & \multicolumn{2}{c|}{ WINTER } \\
\hline$X 1$ & $X^{2} 1$ & $\mathbf{1 6 0}$ & $X^{2} 2$ \\
\hline 120 & $\mathbf{1 4 4 0 0}$ & $\mathbf{1 6 0}$ & $\mathbf{2 5 6 0 0}$ \\
\hline $\mathbf{1 2 0}$ & $\mathbf{1 4 4 0 0}$ & $\mathbf{1 6 0}$ & $\mathbf{2 5 6 0 0}$ \\
\hline $\mathbf{8 0}$ & $\mathbf{6 4 0 0}$ & $\mathbf{1 0 0 0}$ & $\mathbf{1 0 0 0 0 0}$ \\
\hline $\mathbf{8 0 0}$ & $\mathbf{6 4 0 0 0 0}$ & $\mathbf{8 0}$ & $\mathbf{6 4 0 0}$ \\
\hline 40 & $\mathbf{1 6 0 0}$ & $\mathbf{1 0}$ & $\mathbf{1 0 0}$ \\
\hline $\mathbf{1 0}$ & $\mathbf{1 0 0}$ & $\mathbf{6 4 0}$ & $\mathbf{4 0 9 6 0 0}$ \\
\hline $\mathbf{3 2 0}$ & $\mathbf{1 0 2 4 0 0}$ & $\mathbf{8 0}$ & $\mathbf{6 4 0 0}$ \\
\hline 40 & $\mathbf{1 6 0 0}$ & $\mathbf{8 0}$ & $\mathbf{6 4 0 0}$ \\
\hline 40 & $\mathbf{1 6 0 0}$ & $\mathbf{8 0}$ & $\mathbf{6 4 0 0}$ \\
\hline $\mathbf{6 0}$ & $\mathbf{3 6 0 0}$ & $\mathbf{7 0}$ & $\mathbf{4 9 0 0}$ \\
\hline $\mathbf{3 0}$ & $\mathbf{9 0 0}$ & $\mathbf{1 0}$ & $\mathbf{4 0 0 0}$ \\
\hline $\mathbf{8 0}$ & $\mathbf{6 4 0 0}$ & $\mathbf{2 0}$ & $\mathbf{6 4 0 0}$ \\
\hline 40 & $\mathbf{1 6 0 0}$ & $\mathbf{8 0}$ & $\mathbf{6 4 0 0}$ \\
\hline 40 & $\mathbf{1 6 0 0}$ & $\mathbf{8 0}$ & $\mathbf{6 4 0 0}$ \\
\hline $\mathbf{6 0}$ & $\mathbf{3 6 0 0}$ & $\mathbf{8 0}$ & $\mathbf{6 4 0 0}$ \\
\hline $\mathbf{6 0}$ & $\mathbf{3 6 0 0}$ & & \\
\hline
\end{tabular}

\section{DISCUSSION}

\section{Species Used For Different Aliments}

There are about 24 plant species, which are used for different aliments. Ak (calatropis procera) $\mathrm{R}$. Br. is used against skin diseases, eczema, toothache, abdominal pain and asthema (jadhev, 2008a). Harmal (pognum hermella) is used as narcotic, emetic anodyne, hypnotic, anti-lice and fumigated by ladies during small-pox. Dried pulp of bitter fruit of Tumma (citrullus colocynthus) (L.) Shrad. is effective in constipation(Usmanghani, et al., 1997). Fruit of Thumma (citrullus colocynthus) (L.) is useful for the stomach ailments and immunity for Rani Khet diseases and has cold effects against sun-stroke (Heat).

Seeds of Khoob Kalan or chanakhla (sysimbrium irio) Crantz ex steud are used as treatment against Typhoid, small pox. Whole plant of Sonuf (Foeniculum vulgare) 
Miller. is used for digestion problems, gas troubles, female illness and nervous diseases.

Harni Ka Khaj (cistanche tubolose) Wight is effective in diarrhoae and cures sores (Baquar, 1989) and Jawah (carum copticum) Benth. is used as blood purifier. Whole plant of Boophali (corchorus aestuens) Linn. is used for stomach and liver heat. Whole plant of harmal (Pognum hermela) L. is used for the defect in the uterus.

Seeds of Bhakra (Tribulus calendulenisis) L, are used for gallbladder illness and kidney allergy. Whole plant of Boophali (corchorus aestuens) Linn. is used for maleness in man and leucorrhoea.

Leaves of Sumblu or norgundani (vitex negundo) Linn. are used for wounds, oraktsus, and rheumatic pain. Its stem is used for fever. Its juice is useful for gall bladder problems. Its root powder is used for menstrual disorder and restores fertility. If it is roasted seeds powder and wheat flavour is useful for easy delivery.

Neelwat (Withania somnifera) L. is used as an antiinflamatory and sedatve agent (Williamson et. al., 2009). Cooked leaves of Bathu (chenopodium album) L. are used for coronary troubles. Its leaf extract is useful for piles, cough and worms.

Plant decoction of drunk (Polygonum plebijum) R. Br. is used against colic complaints. Plant ash and oil is useful for Eczema. The root of this plant is used in bowel complaints and powdered herb is given in pneumonia ( Trivedi, 2002). Leaf juice of Jau (Hordeum vulgare) Linn. are useful for cataract. Leaves of Jund (Prosopic cineraria) (L.) Druce is useful for leucorrhoea.

Leaf paste and decoction of Mako or Mirchibooti (solanum nigrum) L. is used against jaundice and in case of high fever, cough and liver diseases ( Tridevi, 2002).

Problems related to herbal medicines Business.

1 Pure things are not available.

$2 \quad$ Wild plants are expensive.

3 Hard work and labour is required.

4 Most of area is cultivated.

$5 \quad$ Forests are less, so wild plants have reduced.

$6 \quad$ Wild plants have high prices.

7 People discuss more, the prices of medicines.

8 Pure medicines are not available.

9 Information about plants is negligible.

10 Herbal medicines are shelter-classics Govt. is not paying any attention.

11 Trained people are not enough.

12 Area is being populated.

13 People do not collect plants due to low prices.

14 They insist on purchasing low prices.

People treated per day different places.
Mostly 10 people are treated per day at Adhi kot almost 80 persons are treated per day at Jamali Baluchan. Mostly 20 people are treated per day at Noor pur thal. About 50 people are treated per day at Peeluwance and 20 people are treated at Quluanwala. All classes are treated. 15 people are treated per day. Mostly poor and middle class are treated daily at Biland.

Suggestions to increase the cover of the area.

1. Government should give permission for forest plantation by giving free nurseries.

2. Government should make contact with ther farmers.

3. No of tubewell have to increase.

4. Farmers should be provided with fir nurrsies and plants from the Government nurseries.

\section{Discussion regarding Statistical Analysis and Ethnobotany}

A- Variation in the prices of different fuels yielding species of Khushab District.

Variation in the prices of different fuels yielding species of Khushab District was determined by analysis of variation (ANOVA). Prices vary from Rs.15 to Rs.165 treatments and replicates were made and then total was taken. Sum of square of treatment, sum of square of columns and sum of square of errors were calculated which were $2417,1383,54$ and 980 respectively (table 15).

By using source of variation, sum of square, degree of freedom and mean square, row means, and errors was calculated. Frequency $(\mathrm{f} 1+\mathrm{f} 2)$ was found to know the significance of data. f1 was 2.82 which was the more than actual value i.e. f2 0.165 . So it was found that the variations in prices of different species at that area were significant.

\section{REFERENCES}

[1] Alcorn, J.B. 1984, Development policy forests and peasant farms: reflection on Haustec-managed forests contributions to commercial production and resource conservation, economic botany 38 (4): 3 89-406.

[2] Altieri, M.A. and Merick, L.C. 1987, In situ conservation of crop genetic resources through maintenance of traditional farming system, Economic Botany 4 (!) : 86:96.

[3] Aumeeruddy Y, 1994. Representations to gestion paysannes des agroforeis en kperipherie du pare National Kerinci Seblat a Sumatra, Indonesie, People and Plants working paper no. 3, UNESCO, Paris (English and Spanish version available). 
[4] Balee, W, and Gely, A. 1989. Management of forest succession in Amazonia: the Ka'apor case. In: Posey, D.A. and Balee, W. (eds), "Resource Management in Amazonia: Indigenous and fold strategies", Advances in Economic Botany 7: 129-158.

[5] Baquar, S.R., 1989. Medicinal Plants of Pakistan PP 1489.Cunningham, A.B. 2001. Applied ethnobotany: people, wild plant use and conservation. People and Plants Conservation Series, Earth-scan, London.

[6] Jadhav, D (2008a). Medicined peants of India vol.z. Scientific Publisher, Jodhpur, India. PP.1-269

[7] Johnson, A. 1980. Ethnoecology and planting practices in a swideen agricultural system in: Brokensha D., Warren, D.M. and Werner 0 (eds), Indigenous knowledge systems and development, pp. 49-66. University Press of America, Lanham, Maryland, USA.

[8] Martin, G. 1995, Ethnobotany. A Method Manual,Chapman and Hall, London. Moerman, D.E., R.AW Pemberton, D.Kiefer and Brlin, 1999. A comparative analysis of five medicinal floras. JOURNAL Ethnobotany 19:49-67.

[9] Pei, S.J. and Sajise, P. 1995) Regional study on biodiversity: concepts, frameworks and methods. Yunnan Univeristy Press, China.

[10] Trivedi, P.C. (2002) Ethnobotany. Avishkar Publishers, Distributors, Jaipur, 303003 India. PP. 455.

[11] Usmanghani, K.A. Saeed, A \& Alam, M.T. 1997. Indesyunic Medicine Traditional Medicine Of Herbal, Animal and Mineral Origin in Pakistan. 1-577. University of Karachi.

[12] Williamson, E., Driver, S. and Baxter, K. (2009). Stockley's Herbal Medicines Interactions. Pharmaceutical Press, RPS Publishing, London. 